JÜRGEN APITZ (Hrsg.)

Pädiatrische Kardiologie 


\section{JÜRGEN APITZ}

Herausgeber

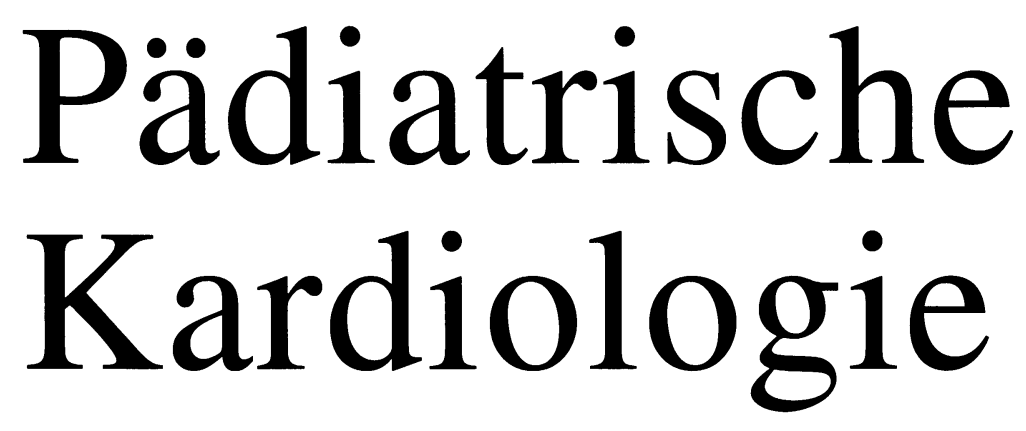

Erkrankungen des Herzens bei Neugeborenen, Säuglingen, Kindern und Heranwachsenden 
Anschrift des Herausgebers:

Prof. Dr. Jürgen Apitz

Lehrstuhl für Kinderkardiologie

Ärztlicher Direktor

der Abteilung Kinderheilkunde II mit Poliklinik

Neuklinikum der Universität

Hoppe-Seyler-Str. 3

72076 Tübingen
Dieses Werk ist urheberrechtlich geschützt. Die dadurch begründeten Rechte, insbesondere die der Übersetzung, des Nachdrucks, des Vortrages, der Entnahme von Abbildungen und Tabellen, der Funksendung, der Mikroverfilmung oder der Verfielfältigung auf anderen Wegen und der Speicherung in Datenverarbeitungsanlagen, bleiben, auch bei nur auszugsweiser Verwertung, vorbehalten. Eine Vervielfältigung dieses Werkes oder von Teilen dieses Werkes ist auch im Einzelfall nur in den Grenzen der gesetzlichen Bestimmungen des Urheberrechtsgesetzes der Bundesrepublik Deutschland vom 9. September 1965 in der Fassung vom 24. Juni 1985 zulässig. Sie ist grundsätzlich vergütungspflichtig. Zuwiderhandlungen unterliegen den Strafbedingungen des Urheberrechtsgesetzes.

(C) 1998 by Springer-Verlag Berlin Heidelberg

Ursprünglich erschienen bei Dr. Dietrich Steinkopff Verlag GmbH \& Co. KG Darmstadt 1998

Softcover reprint of the hardcover 1st edition 1998

Verlagsredaktion: Sabine Ibkendanz - Herstellung: Heinz J. Schäfer

Umschlaggestaltung: Erich Kirchner, Heidelberg

Grafik: B. Baden, Tübingen; P. Lübke, Wachenheim

Die Wiedergabe von Gebrauchsnamen, Handelsnamen, Warenbezeichnungen usw. in dieser Veröffentlichung berechtigt auch ohne besondere Kennzeichnung nicht zu der Annahme, daß solche Namen im Sinne der Warenzeichen- und Markenschutz-Gesetzgebung als frei zu betrachten wären und daher von jedermann benutzt werden dürften.

Produkthaftung: Für Angaben über Dosierungsanweisungen und Applikationsformen kann vom Verlag keine Gewähr übernommen werden. Derartige Angaben müssen vom jeweiligen Anwender im Einzelfall anhand anderer Literaturstellen auf ihre Richtigkeit überprüft werden.

Gesamtherstellung: Konrad Triltsch, Würzburg Gedruckt auf säurefreiem Papier

ISBN 978-3-642-53755-4

ISBN 978-3-642-53754-7 (eBook)
Die Deutsche Bibliothek CIP-Einheitsaufnahme

Pädiatrische Kardiologie : Erkrankungen des Herzens bei Neugeborenen, Säuglingen, Kindern und Heranwachsenden / Jürgen Apitz, Hrsg. Darmstadt : Steinkopff, 1998 


\section{Vorwort}

Die medizinische Fachliteratur ist inzwischen fast unübersehbar geworden. Dennoch fehlt ein neueres deutschsprachiges Buch über Kinderkardiologie. Nach einem Werk, das alle Gebiete der modernen pädiatrischen Kardiologie berücksichtigt, besteht ein echtes Bedürfnis. Der Herausgeber ist deshalb dem Dr. Dietrich Steinkopff Verlag dankbar, daß er bereit war, diese Marktlücke zu schließen.

Die Pädiatrische Kardiologie ist ein relatives junges Spezialgebiet im Bereich der Kinderheilkunde, aber das erste, das eine gewisse Selbständigkeit in größeren Kliniken und eine Anerkennung als Teilgebiet bzw. Schwerpunkt erlangt hat.

Gerade in den letzten Jahren hat die Diagnostik und Behandlung von Herzfehlern im Kindesalter entscheidende Veränderungen erfahren. In der Diagnostik haben die nichtinvasiven Untersuchungsmethoden die invasiven erheblich zurückgedrängt. In der Behandlung haben die interventionellen Maßnahmen (mit invasivem $\mathrm{Zu}$ gang) ständig an Bedeutung gewonnen. In der operativen Behandlung hat sich die bereits vor Jahren vom Herausgeber geforderte Frühkorrektur durchgesetzt. Für nicht korrigierbare Herzfehler stehen modifizierte kreislauftrennende Operationen, inzwischen mit relativ geringem Risiko, und Transplantationen zur Verfügung.

Verlag und Herausgeber haben sich bemüht, das gesamte Wissensgebiet der pädiatrischen Kardiologie auf dem neuesten Stand in einem Band zusammenzufassen. Um ein zügiges Erscheinen dieses Werkes zu ermöglichen, war es notwendig, das umfangreiche Stoffgebiet aufzuteilen. Neben Mitarbeitern der eigenen Abteilung wurden weitere erfahrene Kinderkardiologen als Autoren gewonnen. Jeder der Autoren ist durch seine Aufgaben in der Lehre, Forschung und Krankenversorgung zeitlich ausgelastet. Die Mitarbeit an diesem Buch stellte damit eine zusätzliche Belastung dar. Um so mehr bin ich allen Autoren für die aktive Mitarbeit an diesem Werk und für ihre erfreulich gute Zusammenarbeit dankbar.

Die vorgegebenen Seitenzahlen hatten zwangsläufig auch eine Begrenzung der Literaturangaben und der Abbildungen zur Folge. In der ergänzenden Literatur wird auf weitere Publikationen hingewiesen. Wer auf einem bestimmten Gebiet der Pädiatrischen Kardiologie oder ihrer Grenzgebiete forschen und publizieren will, muß ohnehin die einschlägige Literatur und die entsprechenden Journale selbst erarbeiten.

Die verwendete Nomenklatur ist international gebräuchlich. Sicher gibt es auf einzelne Fragen individuell unterschiedliche Antworten, so die Indikation zu invasiven diagnostischen oder thera- 
peutischen Maßnahmen oder die Indikation zu palliativen oder korrektiven Operationen sowie über den Zeitpunkt dieser Eingriffe. Der Herausgeber und einige Autoren sind gleichzeitig Mitglieder der Leitlinien- und Konsensuskonferenz der Deutschen Gesellschaft für Pädiatrische Kardiologie und haben die Leitlinien dieser Gesellschaft für die diagnostischen und therapeutischen Maßnahmen bei angeborenen und erworbenen Herzfehlern im Kindesalter erarbeitet. Die in dem vorliegenden Werk niedergelegten Richtlinien stimmen mit den Leitlinien der Deutschen Gesellschaft für Pädiatrische Kardiologie überein, so daß dieses Buch zugleich das erste ist, das diese Leitlinien berücksichtigt. Dadurch ist es auch gelungen, u.U. gegensätzliche Meinungen auszugleichen und - wie ich hoffe - ein einheitliches Werk zu schaffen.

In der schriftlichen Wiedergabe der Geräusche und ihrer Lokalisation wurde die von den einzelnen Autoren angegebene Bezeichnung nicht verändert; sie ist nach den jeweiligen Schulen nicht ganz einheitlich. Die Wiedergabe seitlicher Röntgen- und Angiokardiographieaufnahmen wird in einzelnen Kliniken, aber auch in Lehrbüchern, unterschiedlich gehandhabt. Es wurde hier generell die Darstellungsform gewählt, bei der „,dorsal“ bzw. ,posterior“ links im Bild und ,ventral“" bzw. „,anterior“ rechts im Bild erscheint.

Das Werk enthält viele Abbildungen von Angiokardiogrammen, um das Dargestellte zu illustrieren. Es handelt sich bei vielen Angiokardiogrammen um ältere Aufnahmen, aus einer Zeit, in der die moderne Echokardiographie zumindest mit dem heutigen Auflösungsvermögen noch nicht zur Verfügung stand. In einigen Abbildungen wurde das Aufnahmedatum vermerkt. Viele dieser Aufnahmen sind heute durch die Möglichkeiten der nichtinvasiven Diagnostik durch die Echokardiographie und Kernspintomographie nicht mehr indiziert.

Autoren, Verlag und Herausgeber haben alle Anstrengungen unternommen, um die angegebenen Dosierungen von Medikamenten korrekt und in den üblichen Standards wiederzugeben. Der Leser ist jedoch dringend aufgefordert und auch verpflichtet, vor der Anwendung jedes Medikamentes die Dosierungsanweisungen zu überprüfen sowie Kontraindikationen und Unverträglichkeiten mit anderen Arzneimitteln zu beachten.

Es ist mir ein besonderes Anliegen, allen Autoren für ihre freundliche und umgehende Mitarbeit zu danken. Die Kooperation der Autoren hat die nicht unerhebliche Arbeit des Herausgebers erleichtert. Dem Dank an die Autoren möchte ich den Dank an den Dr. Dietrich Steinkopff Verlag, Darmstadt, anschließen, hier besonders Frau S. Ibkendanz und Frau D. Wolf-Hollenbach, die sich mit großem Engagement diesem Werk gewidmet haben, und an Frau H. Schoeler, der das Werk seine sprachliche Qualität und didaktische Struktur maßgeblich verdankt. Dank gebührt auch Frau B. Baden für ihre unermüdliche Arbeit bei der Anfertigung der zahlreichen Abbildungen. 
Mein besonderer Dank gilt meiner Sekretärin Frau E. Dessecker, die neben der täglichen Arbeit nicht nur den umfangreichen Schriftverkehr für dieses Buch bewältigen mußte, sondern auch die vom Herausgeber verfaßten Kapitel.

Schließlich möchte ich auch meinen ärztlichen Mitarbeitern danken, die mich in der Zeit intensiver Autoren- und Herausgeberarbeit in meiner klinischen Tätigkeit entlastet haben.

Der Dr. Dietrich Steinkopff Verlag hat es ermöglicht, relativ viele Abbildungen mit farbiger Unterlegung und Vierfarbabbildungen zu verwenden. Dies kommt der Didaktik und dem Verständnis des Textes zugute. Dabei ist das Werk weniger für den studentischen Unterricht gedacht, sondern vielmehr für die Weiterbildung im Teilgebiet bzw. Schwerpunkt Kinderkardiologie. Es soll in umfassender und dennoch knapper handlicher Form dem in der Klinik und Praxis tätigen Kinderarzt und Kinderkardiologen als Nachschlagewerk und damit als zuverlässiger Ratgeber dienen. Das umfangreiche Sachregister läßt eine schnelle Orientierung über alle Fragen der Pädiatrischen Kardiologie und damit auch über den gegenwärtigen Stand der Diagnostik und Therapie angeborener und erworbener Herzfehler zu. Dabei hat das Werk seinen Schwerpunkt in der Diagnostik und in der interventionellen Therapie. Bei der Beschreibung der Therapie werden auch die operativen Möglichkeiten berücksichtigt, wobei die Darstellung der einzelnen Operationsverfahren nicht das primäre Anliegen dieses Buches sein kann. Hierfür gibt es spezielle Operationslehren, aber auch Werke über die Operationen angeborener und erworbener Herzfehler im Kindesalter. Der präund postoperativen Intensivbehandlung ist nur ein begrenzter Raum gewidmet; eine ausführlichere Darstellung würde den vorgesehenen Rahmen dieses Buches überschreiten.

So hoffe ich, daß das Buch alle Voraussetzungen erfüllt, um von einer breiteren Leserschaft akzeptiert zu werden. Schon die vor der Drucklegung zu beobachtende Nachfrage läßt hoffen, daß das Werk auf großes Interesse in Fachkreisen stößt. 


\section{Inhaltsverzeichnis}

Vorwort $\ldots \ldots \ldots \ldots \ldots \ldots \ldots \ldots \ldots \ldots \ldots, \mathrm{V}$

Einführung $\ldots \ldots \ldots \ldots \ldots \ldots \ldots \ldots \ldots \ldots \ldots \ldots \ldots$

$1.1 \quad$ Geschichte der pädiatrischen Kardiologie (J. APITZ) . . . . . 1

1.2 Häufigkeit angeborener und erworbener Herzfehler, Letalität und natürlicher Verlauf angeborener Herzfehler (J. APITZ) $\ldots \ldots \ldots \ldots \ldots \ldots \ldots \ldots \ldots \ldots \ldots .4$

1.3 Spezielle Probleme des Neugeborenenalters

(Chr. Irtel von Brenndorff, J. APITZ) . . . . . . . . . 10

$1.4 \quad$ Versorgung herzkranker Kinder in der

Bundesrepublik Deutschland (J. APITZ) ............ 14

1.5 Embryologische Entwicklung des Herzens und Störungen

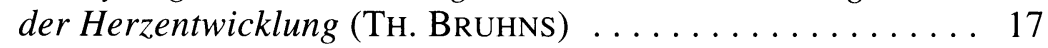

1.5.1 Herz ............................. 17

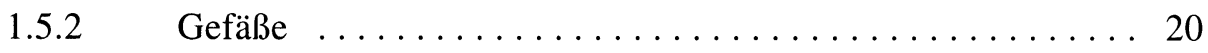

1.6 Ätiologie angeborener Herzfehler und genetische Beratung

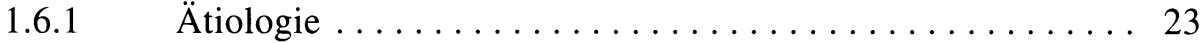

1.6.2 Genetische Beratung $\ldots \ldots \ldots \ldots \ldots \ldots \ldots \ldots \ldots \ldots$

$1.7 \quad$ Fetale Zirkulation und postnatale Veränderungen des Kreislaufs (E. Steil, G. Mielke) ............... 31

1.7.1 Anatomie und Physiologie der fetalen Zirkulation ....... 31

1.7.2 Die perinatale Bedeutung des Foramen ovale .......... 32

1.7.3 Die Rolle des fetalen Ductus arteriosus . . . . . . . . . . . 32

1.7.4 Der Ductus venosus . . . . . . . . . . . . . . . . 33

1.7.5 Grenzen der Beurteilung der fetalen Kreislaufsituation und indirekte diagnostische Hinweise ............... 34

1.7.6 Der physiologische Übergang von der fetalen zur neonatalen Zirkulation ..................... 34

1.7.7 Störungen der perinatalen Kreislaufumstellung: persistierende fetale Zirkulation und persistierende pulmonale Hypertonie . . . . . . . . . . . . . . . . . . . . . . 34

2 Untersuchungsmethoden .................. 37

$2.1 \quad$ Anamnese, klinische Untersuchung (J. APITZ) . ......... 37

2.1.1 Anamnese ........................... 37 


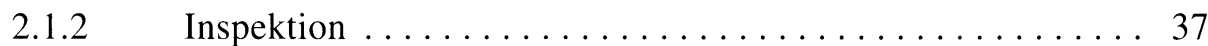

2.1.3 Palpation .............................. 38

2.1.4 Perkussion .............................. 39

2.1.5 Auskultation ........................... 39

$2.2 \quad$ EKG, Vektorkardiogramm, Phonokardiogramm (J. APITZ) . . 45

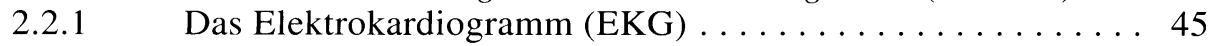

2.2.2 Vektorkardiogramm (VCG) . . . . . . . . . . . . . . . 69

2.2.3 Das Phonokardiogramm (PKG) $\ldots \ldots \ldots \ldots \ldots \ldots \ldots$

2.3 Langzeit-EKG, (Langzeit-)Blutdruckmessung

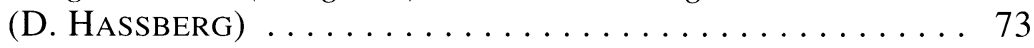

2.3.1 Langzeit-Elektrokardiographie $\ldots \ldots \ldots \ldots \ldots \ldots \ldots 73$

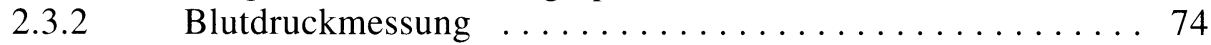

2.3.3 Langzeit-Blutdruckmessung (24-h-Blutdruckmessung) $\ldots 75$

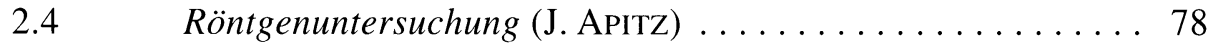

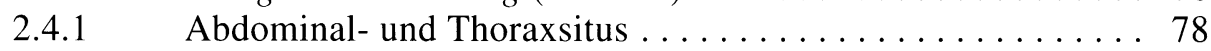

2.4.2 Herzlage . . . . . . . . . . . . . . . . . . . . . 79

$2.4 .3 \quad$ Herzgröße $\ldots \ldots \ldots \ldots \ldots \ldots \ldots \ldots \ldots \ldots \ldots \ldots \ldots$

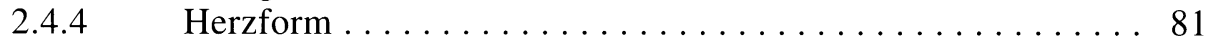

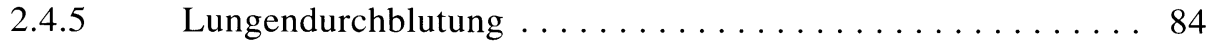

2.4.6 Anomalien des knöchernen Thorax ................ 86

$2.5 \quad$ Kernspintomographie, MR-Spektroskopie (L. SIEVERDING) . 88

2.5.1 Kernspintomographie ................... 88

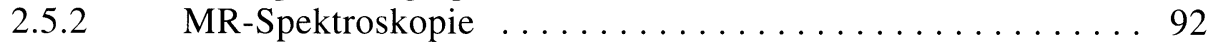

$2.6 \quad$ Echokardiographie (H. BARTH) $\ldots \ldots \ldots \ldots \ldots \ldots 9$

2.6.1 Geschichtliches ........................... 98

2.6.2 M-mode-Echokardiographie ................ 98

2.6.3 Zweidimensionale Echokardiographie (2D) $\ldots \ldots \ldots \ldots 98$

2.6.4 Kontrastechokardiographie .................. 101

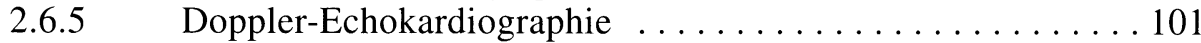

2.7 Transösophageale Echokardiographie (TEE) (H. BARTH) . . 106

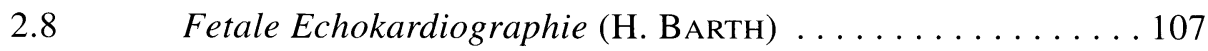

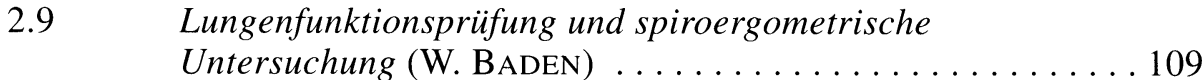

$2.10 \quad$ Invasive Herzdiagnostik (J. APITZ) . . . . . . . . . . . . . 114

$2.10 .1 \quad$ Geschichtliches ........................... 114

2.10.2 Herzkatheterisierung . . . . . . . . . . . . . . . 115

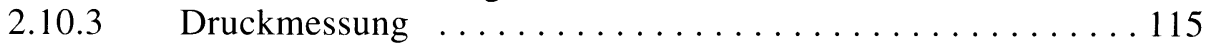

$2.10 .4 \quad$ Vektorielle Druckschreibung ................... 116

2.10.5 Bestimmung der Sauerstoffsättigung . . . . . . . . . . . . . . 117

2.10.6 Berechnung von Kreislaufgrößen . . . . . . . . . . . . 118

2.10.7 Ergänzende invasive Untersuchungen . . . . . . . . . . . . 119

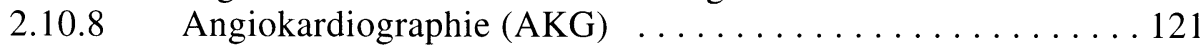

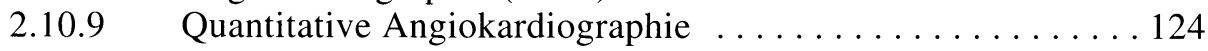


2.10.10 Komplikationen und Risiko der invasiven

Untersuchungsmethoden ................... 125

2.10 .11 Einwilligung zur invasiven Diagnostik . . . . . . . . 126

2.10 .12 Indikationen für die invasive Diagnostik $\ldots \ldots \ldots \ldots \ldots 127$

2.10 .13 Invasive Intensivüberwachung $\ldots \ldots \ldots \ldots \ldots \ldots \ldots 128$

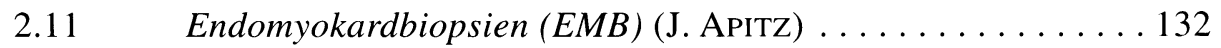

2.12 Elektrophysiologische Untersuchungen (Тн. TEUFEL) . . . 135

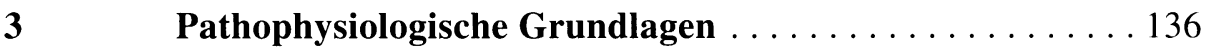

3.1 Physiologische Vorbemerkungen (CHR. APITZ, J. APITZ) . . . 136

3.2 Pathophysiologie der Herzfehler ohne Shunt

(J. ApITZ, Chr. ApITZ) . . . . . . . . . . . . . . . 137

3.2.1 Druck- und Volumenbelastungen des rechten Herzens . . . . 138

3.2.2 Druck- und Volumenbelastungen des linken Herzens ....... 141

3.3 Pathophysiologie der Links-Rechts-Shunts

(Chr. ApITZ, J. APITZ) . . . . . . . . . . . . . . . . . . . . . . 145

3.3.1 Links-Rechts-Shunts auf Ventrikelebene ............ 146

3.3.2 Links-Rechts-Shunts im Bereich der großen Gefäße . . . . . . 147

3.3.3 Links-Rechts-Shunts auf Vorhofebene

(einschließlich partiell fehlmündender Lungenvenen) . . . . 148

3.4 Pathophysiologie der Rechts-Links-Shunts

(J. APITZ, Chr. APITZ) . . . . . . . . . . . . . . . . . . . . 149

3.4.1 Physiologischer Rechts-Links-Shunt . . . . . . . . . . . . . 149

3.4.2 (Fixierte) Pulmonale Hypertonie . . . . . . . . . . . . . . . 149

3.4.3 Rechts-Links-Shunt auf Ventrikelebene ............ 153

3.4.4 Rechts-Links-Shunt auf Gefäßebene . ............ 153

3.4.5 Rechts-Links-Shunt auf Vorhofebene . . . . . . . . . 155

3.4.6 Rechts-Links-Shunt auf der Ebene der zuführenden Gefäße . 156

3.5 Pathophysiologie bei Klappenatresien (J. APITZ, CHR. APITZ) 156

3.6 Hypoxämie und ihre Komplikationen (J. APITZ, CHR. APITZ) .157

3.7 Pathophysiologie der Herzinsuffizienz (CHR. APITZ, J. APITZ) 162

$4 \quad$ Anomalien des linken Ventrikels und der Aorta . . . . . . . 169

$4.1 \quad$ Auswurfbehinderungen des linken Ventrikels (W. BADEN) . . 169

4.1.1 Valvuläre Aortenstenose . . . . . . . . . . . . . . . . 169

4.1.2 Bikuspide Aortenklappe ........................ 174

4.1.3 Subvalvuläre Aortenstenose . . . . . . . . . . . . . . . 174

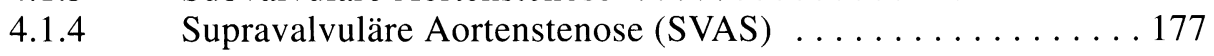

4.2 Hypertrophe Kardiomyopathie (A. A. SCHMALTZ) . . . . . 181

4.3 Anomalien des Aortenbogens und des Aortenisthmus

(W. BADEN) . . . . . . . . . . . . . . . . . . . . . . . 185

4.3.1 Aortenisthmusstenose und Aortenbogenstenose .......... 185 
4.3.2 Kongenitaler Gefäßring, doppelter Aortenbogen, A. lusoria . . 194

4.3.3 Unterbrochener Aortenbogen . . . . . . . . . . . . . 197

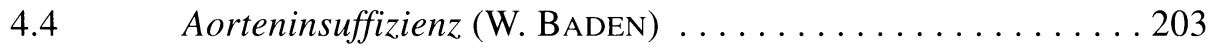

$4.5 \quad$ Kongenitale Mitralklappenanomalien (A. A. SchMALTZ) . . . 207

4.5.1 Mitralklappenstenose ...................... 207

4.5.2 Mitralklappeninsuffizienz . . . . . . . . . . . . . . . . . 209

4.5.3 Mitralklappenprolaps ................. 210

$5 \quad$ Anomalien des rechten Ventrikels

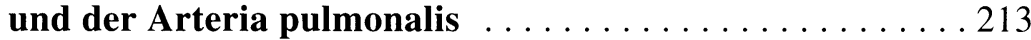

5.1 Auswurfbehinderungen des rechten Ventrikels (G. WAGNER) . 213

5.1.1 Valvuläre Pulmonalstenose ................. 213

5.1.2 Isolierte Infundibularstenose . . . . . . . . . . . . . . . . . . . 220

5.1.3 Zweikammriger rechter Ventrikel ............. 221

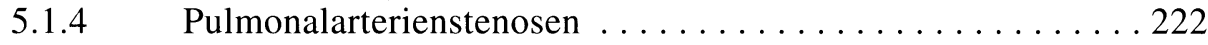

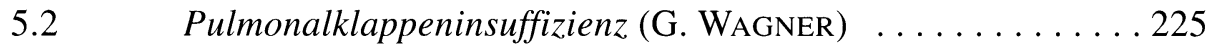

$5.3 \quad$ Kongenitale Trikuspidalklappenstenose

und-insuffizienz (J. APITz) . . . . . . . . . . . . . . . . 229

5.3.1 Die kongenitale Trikuspidalklappeninsuffizienz . . . . . . . . . 229

5.3.2 Die kongenitale Trikuspidalklappenstenose ......... . 230

6 Angeborene Anomalien der Lungengefäße . . . . . . . . . 232

6.1 Fehlabgang einer Pulmonalarterie aus der Aorta (Hemitruncus) (J. APITZ, CHR. APITZ) . . . . . . . . . . 233

$6.2 \quad$ Pulmonalisschlinge (J. APITZ, CHR. APITZ) . . . . . . . . 236

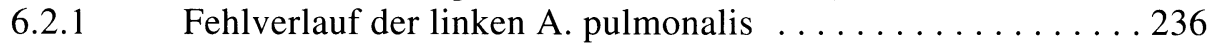

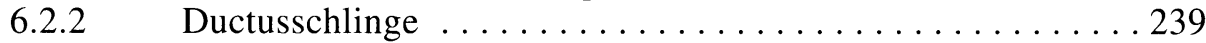

6.3 Angeborene (arteriovenöse) pulmonale

Kurzschlußverbindungen einschließlich der a.v.-Aneurysmen der Lunge (J. ApITz, CHR. APITz) . . . . . . . . . . . 241

6.3.1 Normale angeborene arteriovenöse Kurzschlußverbindungen der Lunge . . . . . . . . . . . . . . . . . . . . . 241

6.3.2 Pathologische angeborene arteriovenöse Kurzschlußverbindungen der Lunge, die sog. arteriovenöse Lungenfistel 242

$6.4 \quad$ Einseitige Lungenagenesie, -aplasie oder-hypoplasie (J. ApITZ, Chr. APITZ) _. . . . . . . . . . . . . . . . . 245

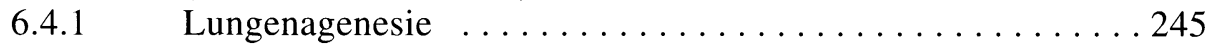

6.4.2 Lungenhypoplasie . . . . . . . . . . . . . . 247

6.5 Systemopulmonale Gefäßverbindungen (Anastomosen) (J. ApITZ, Chr. ApITz) . . . . . . . . . . . . . . . . . . . . 249

6.5.1 Lungendurchblutung bei einseitigem Fehlen einer Pulmonalarterie . . . . . . . . . . . . . . . . . . . . 249

6.5.2 Lungendurchblutung bei Pulmonalatresie . . . . . . . . . . . 249 
$6.5 .3 \quad$ Lungensequester . . . . . . . . . . . . . . 251

6.5.4 Systemopulmonale Aneurysmen . . . . . . . . . . . 254

$7 \quad$ Angeborene Herzfehler mit überwiegendem

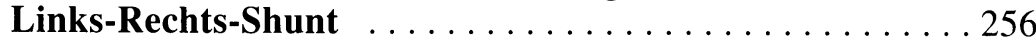

$7.1 \quad$ Links-Rechts-Shunts auf Vorhofebene (A. A. Schmaltz) . . . 256

7.1.1 Vorhofseptumdefekte .................... 256

7.1.2 Partielle Lungenvenenfehlmündung . . . . . . . . . . . . . . . 260

7.1.3 Pulmonalvenenstenose, Cor triatriatum . . . . . . . . . . . . 263

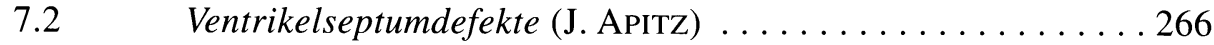

7.3 Endokardkissendefekte - AV-Septumdefekte (AVS)

(H. SINGER) . . . . . . . . . . . . . . . . . . . 279

7.4 Ductus arteriosus persistens und aortopulmonales Fenster

7.4.2 Aortopulmonales Fenster . . . . . . . . . . . . . . . . . . 297

7.5 Arteriovenöse Fisteln des Systemkreislaufs (G. WAGNER) . . . 301

8 (Komplexe) Angeborene Herzfehler

mit überwiegendem Rechts-Links-Shunt . . . . . . . . . . . 304

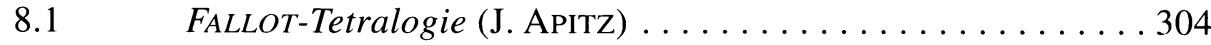

8.2 Pulmonalatresie mit Ventrikelseptumdefekt (VSD)

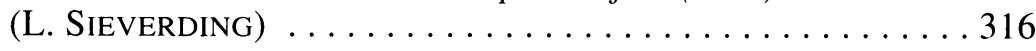

8.3 Ursprung beider Gefäße aus einem Ventrikel

(partielle Transposition der großen Gefäße)

8.3.1 Double outlet right ventricle $\ldots \ldots \ldots \ldots \ldots \ldots \ldots \ldots \ldots . \ldots \ldots$

8.3.2 Double outlet left ventricle . . . . . . . . . . . . . . . . . 329

8.4 Komplette Transposition der großen Arterien

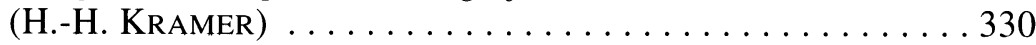

8.5 Angeboren korrigierte Transposition der großen Arterien

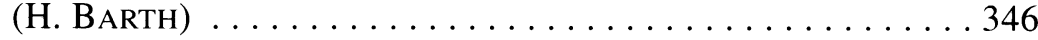

8.6 Truncus arteriosus communis (J. APITZ) . . . . . . . . . . . . 349

8.7 Trikuspidalatresie (J. VoGT) . . . . . . . . . . . . . . . . 359

$8.8 \quad$ Pulmonalatresie mit intaktem Ventrikelseptum (J. VoGT) . . . 362

8.9 Das sogenannte univentrikuläre Herz (H. BARTH) . . . . . 366

8.10 Mitralatresie (Atresie der linken Atrioventrikularklappe)

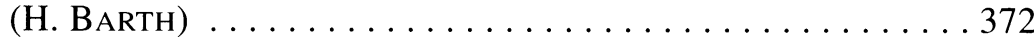

8.11 Hypoplastisches Linksherzsyndrom (HLHS) mit Aorten-

und/oder Mitralatresie (J. APITZ) . . . . . . . . . . . . . 374 
8.12 Total fehlmündende Lungenvenen (H. E. Ulmer) . . . . . . . 381

8.13 Anomaler Systemvenenrückfluß (Hohlvenenanomalien)

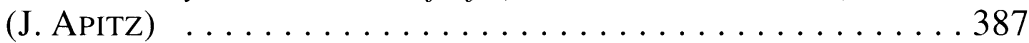

8.13.1 Hohlvenenanomalien mit Mündung in den rechten Vorhof . . . 388

8.13.2 Fehlmündung von Systemvenen in den linken Vorhof . . . . . 391

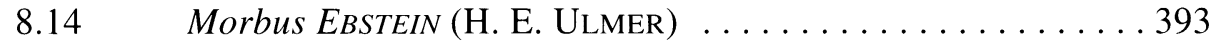

8.15 Primäre und sekundäre pulmonale Hypertonie (J. BREUER) . . 399

9 Lageanomalien des Herzens (H. BARTH) . . . . . . . . 406

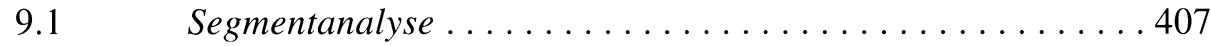

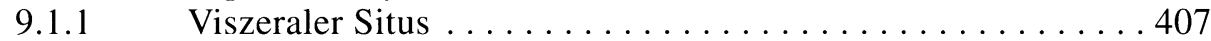

9.1.2 Atrialer Situs . . . . . . . . . . . . . . . . . . . . . . 408

9.1.3 Die atrioventrikulären Verbindungen ............. 408

9.1.4 Ventrikulärer Situs ..................... . 409

9.1.5 Die ventrikuloarteriellen Verbindungen ............ 409

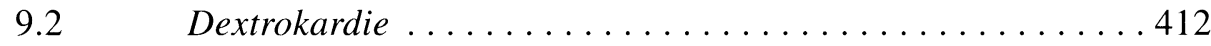

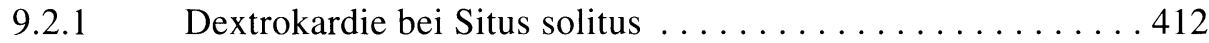

9.2.2 Dextrokardie bei Situs inversus . . . . . . . . . . 413

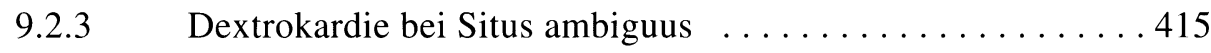

$9.3 \quad$ Mesokardie ....................... 415

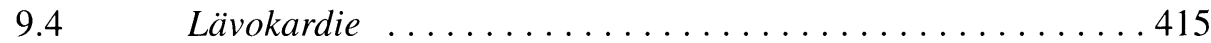

9.5 „Criss-cross“-atrioventrikuläre Verbindungen,

9.6 Prinzipien der Diagnostik kongenitaler Lageanomalien des Herzens . . ...................416

9.7 Prinzipien der Therapie kongenitaler Lageanomalien des Herzens . . .................... 417

$9.8 \quad$ Angeborene Perikarddefekte . . . . . . . . . . . . . 417

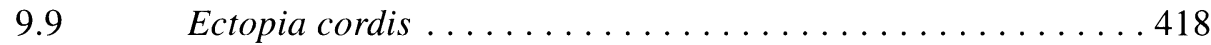

10 Anomalien der Koronararterien . . . . . . . . . . . . . 419

$10.1 \quad$ Angeborene Anomalien der Koronararterien (J. APITZ) . . . . 419

10.1.1 Anomalien mit Ursprung der Koronararterien aus der Aorta . . 419

10.1.2 Ursprung einer Koronararterie aus der A. pulmonalis . . . . . 424

10.2 Erworbene Erkrankungen der Koronararterien (J. ApITZ, Chr. ApITZ) . . . . . . . . . . . . . . . . . . . 429

11 Seltenere angeborene und erworbene Anomalien

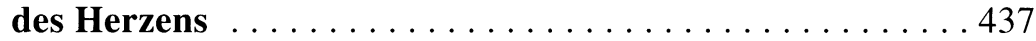

$11.1 \quad$ Endokardfibroelastose (A. A. Schmaltz) . . . . . . . . 437

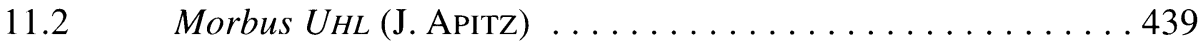


11.3 Divertikel und Aneurysmen des linken und rechten Ventrikels

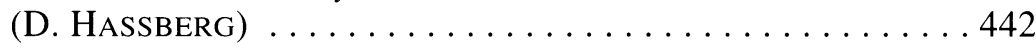

11.4 Herztumoren (L. SIEVERDING) . . . . . . . . . . . . 444

11.4.1 Rhabdomyome ....................... . 447

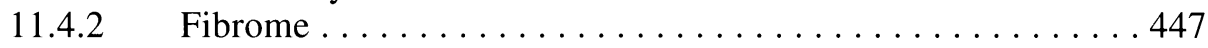

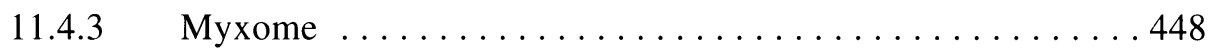

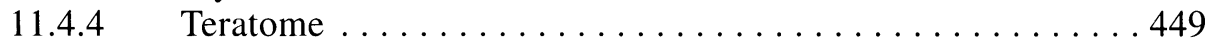

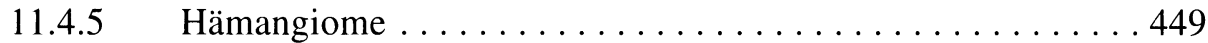

11.4 .6 Maligne Tumoren . . . . . . . . . . . . . . . . . 449

11.4.7 Sekundäre Tumoren . . . . . . . . . . . . . . . 450

12 Systemerkrankungen mit Herzbeteiligung $\ldots \ldots \ldots \ldots 45$

$12.1 \quad$ Metabolische Erkrankungen (L. SIEVERDING) . . . . . . . 453

12.1.1 Lysosomale Speichererkrankungen . . . . . . . . . . . 453

12.1.2 Glykogenspeichererkrankungen .............. 453

12.1.3 Mitochondriopathien ................... 454

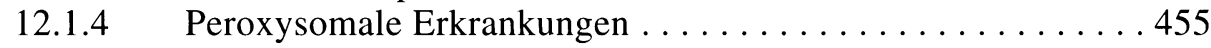

12.2 Neuromuskuläre Erkrankungen (L. SIEVERDING) . . . . . . . 455

12.2.1 Muskeldystrophien .................... 455

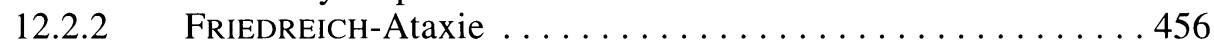

12.3 Endokrine Erkrankungen (L. SIEVERDING) . . . . . . . . . 456

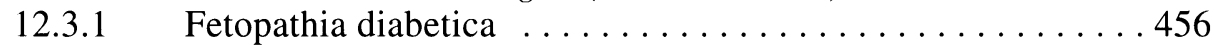

12.3.2 Hypothyreose ................... 456

12.3.4 Hyperthyreose . . . . . . . . . . . . . . 456

12.4 Erkrankungen des Bindegewebes (L. SIEVERDING) . . . . . . . 457

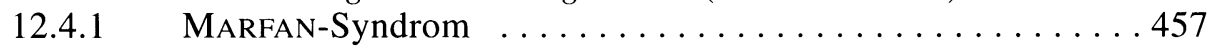

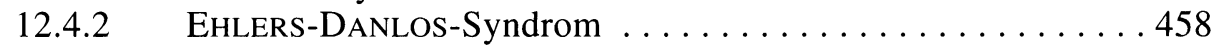

13 Entzündliche Erkrankungen des Herzens,

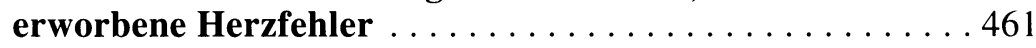

13.1 Nichtrheumatische entzündliche Herzerkrankungen -

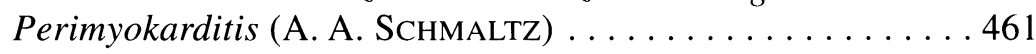

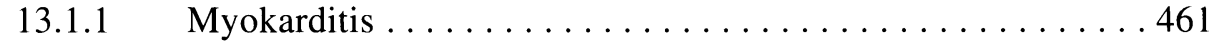

13.1.2 Isolierte Perikarditis . . . . . . . . . . . . . . 464

13.2 Dilatative Kardiomyopathie (A. A. Schmaltz) . . . . . . . 466

13.3 Akute und chronische rheumatische Entzündungen des Herzens, rheumatische Herzfehler (H. NETZ) . . . . . . . . 469

13.3.1 Rheumatisches Fieber .................... 469

13.3.2 Chronische rheumatische Herzerkrankung . . . . . . . . . . 475

13.4 Die infektiöse (bakterielle) Endokarditis im Kindesund Jugendalter (H.-H. KRAMER) . . . . . . . . . . . . . 480

14 Herzrhythmusstörungen . . . . . . . . . . . . . . 489

14.1 Diagnostik und Therapie von Herzrhythmusstörungen

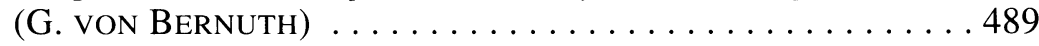


14.1.1 Normale Herzfrequenz im Kindesalter . . . . . . . . . . . . . 489

14.1.2 Pathophysiologie der Herzrhythmusstörungen . . . . . . . . . 490

14.1.3 Diagnostik der Herzrhythmusstörungen . . . . . . . . . 492

14.1.4 Formen von Herzrhythmusstörungen . . . . . . . . . . . . . . 492

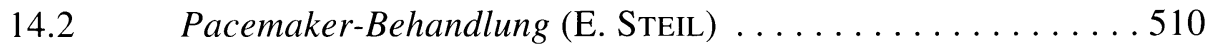

15 Spezielle kinderkardiologische Probleme . . . . . . . . . 519

15.1 Unschuldige Herz- und Gefäßgeräusche im Kindesalter (J. APITZ) . . . . . . . . . . . . . . . . . . . . . . 519

15.2 Funktionelle Störungen des kardiovaskulären Systems

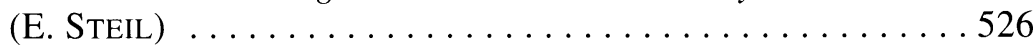

15.3 Langzeitergebnisse nach Herzoperationen (E. STEIL) . . . . . 532

15.4 Die Betreuung von Jugendlichen, Heranwachsenden und Erwachsenen mit angeborenen Herzfehlern Sport bei herzkranken Kindern - Rehabilitation

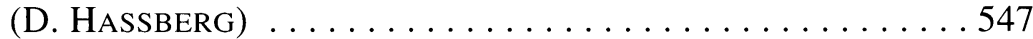

15.4.1 Die Betreuung von Jugendlichen, Heranwachsenden und Erwachsenen mit angeborenen Herzfehlern . . . . . . 547

15.4.2 Sport bei herzkranken Kindern . . . . . . . . . . . . . 548

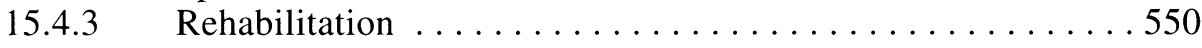

15.5 Psychosoziale Aspekte von Patienten mit angeborenen Herzfehlern und ihren Familien

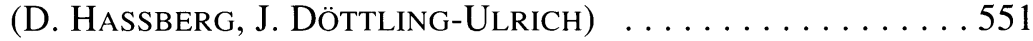

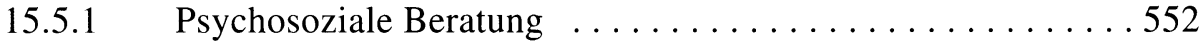

15.5.2 Das Konzept der familienorientierten Nachsorge . . . . . . . 553

$15.6 \quad$ Hypertonie des Systemkreislaufs (W. RoSENDAHL) . . . . . . . 555

15.7 Nephrologische Probleme bei Kindern mit angeborenen und erworbenen Herzfehlern (W. RosENDAHL) . . . . . . . . 559

15.7.1 Störungen der Nierenfunktion bei Herzinsuffizienz . . . . . . 559 15.7.2 Begleitende Nierenerkrankungen . . . . . . . . . . . 560

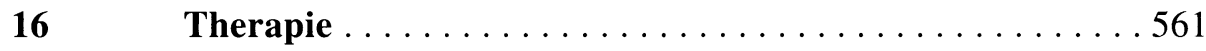

16.1 Prinzipien der medikamentösen Behandlung -

Therapie der Herz-Kreislauf-Insuffizienz (E. STEIL) . . . . . . 561

16.1.1 Pathophysiologische Ansatzpunkte der medikamentösen kardialen Therapie ................... 561

16.1.2 Medikamentengruppen bei der Behandlung

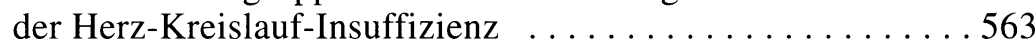

16.2 Interventionskardiologie (L. SiEverding, J. APITZ) . . . . . . 568

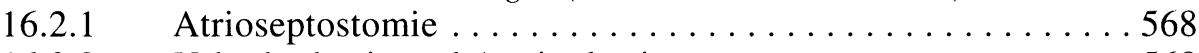

16.2.2 Valvuloplastie und Angioplastie ............... 568

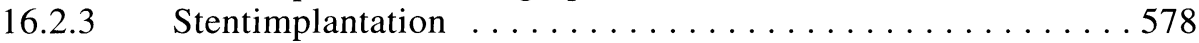

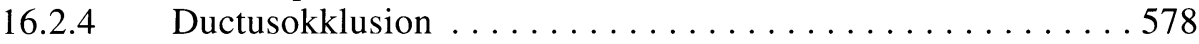

16.2.5 ASD-Verschluß . . . . . . . . . . . . . . . . . . . 579 


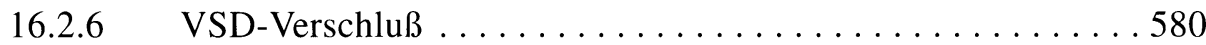

16.2.7 Gefäßverschlüsse $\ldots \ldots \ldots \ldots \ldots \ldots \ldots \ldots \ldots \ldots \ldots \ldots$

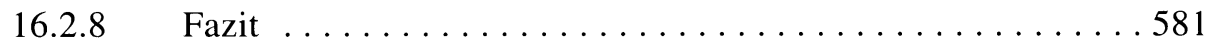

16.3 Prinzipien der chirurgischen Behandlung

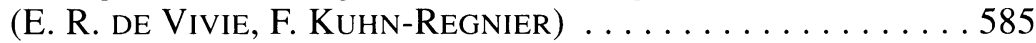

16.3.1 Vorbemerkungen ...................... 585

16.3.2 Zugangswege zum Herzen und zu den herznahen

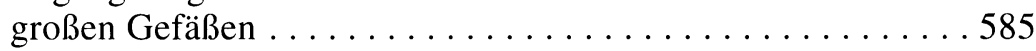

16.3.3 Physiologie und Technik der extrakorporalen Zirkulation . . . 585

16.3.4 Grundlagen der Myokardprotektion . . . . . . . . . . . 586

16.3.5 Palliative Operationen ...................... . 587

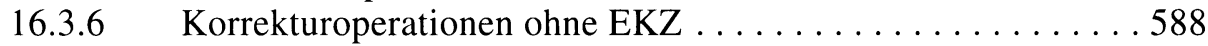

16.3.7 Korrekturoperationen mit EKZ . . . . . . . . . . . . . 590

16.3.8 Defekte des Vorhof- und Kammerseptums einschließlich der AV-Klappen . ....................... 592

16.3.9 Operative Strategien bei kombinierten und komplexen angeborenen Herzfehlern . . . . . . . . . . . . . . . . . . . . 594

$16.4 \quad$ Herz- und Lungentransplantation im Kindesalter

(F. KUHN-RegnieR, E. R. DE VIVIE) . . . . . . . . . . . . 600

16.4.1 Herztransplantation ........................6 600

16.4.2 Lungentransplantation .....................6 601

16.4.3 Herz-Lungentransplantation . .................6 603

$16.5 \quad$ Postoperative Intensivbehandlung und postoperative Probleme (E. STEIL) . . . . . . . . . . . . 604

16.6 Behandlung der postoperativen pulmonalen Hypertonie (J. BREUER) . . . . . . . . . . . . . . . . . . . . . . . 610

16.7 Kinderkardiologische Notfälle und ihre Behandlung,

Wiederbelebung und Reanimation (E. STEIL, W. BADEN) . . . 616

16.7.1 Prähospitale und intrahospitale Vorbeugung ...........616 616

16.7.2 Vitienspezifische Gefahren und Notfälle ..............6 616

16.7.3 Digitalisintoxikation .......................6. 620

16.7.4 Hyperkaliämie . ......................6. 620

16.7.5 Weitere kinderkardiologische Notfallsituationen ......6621

16.7.6 Prinzipien der Reanimation bei kardiologischen Notfällen im Säuglings- und Kindesalter . . . . . . . . . . . . 622

16.7.7 Defibrillation und Behandlung der elektromechanischen Dissoziation .........................6624

16.8 Schock und Schockbehandlung -

Störungen der Mikrozirkulation (E. STEIL, W. BADEN) . . . . . 626

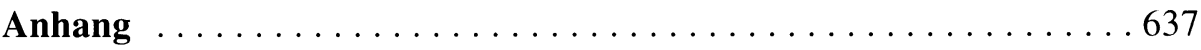

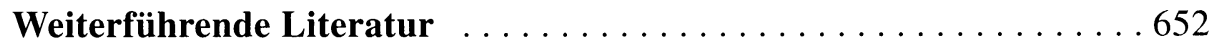

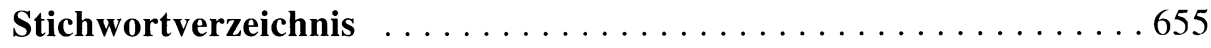




\section{Anschriften der Verfasser}

Dr. med. Christian Apitz

Univ.-Kinderklinik Homburg/Saar, 66421 Homburg/Saar, Oskar-Orth-Straße

Prof. Dr. med. Jürgen Apitz

Lehrstuhl für Kinderkardiologie,

Ärztlicher Direktor der Abteilung Kinderheilkunde II mit Poliklinik (Schwerpunkte: Kardiologie, Nephrologie, Pulmologie, Intensivbehandlung), Neuklinikum der Universität, 72076 Tübingen, Hoppe-Seyler-Str. 3

Dr. med. Winfried Baden

Oberarzt der Abt. Kinderheilkunde II. Universitätskinderklinik, Neuklinikum der Universität, 72076 Tübingen, Hoppe-Seyler-Str. 3

Dr. med. Helmut Barth

Oberarzt, Zentrum für Kinderheilkunde der Philipps-Universität, Univ.-Kinderklinik, 35033 Marburg, Deutschhausstr. 12

Prof. Dr. med. Götz von Bernuth

Direktor der Klinik für Kinderkardiologie Univ.-Klinikum der Rhein.-Westf.-Techn. Hochschule Aachen, 52074 Aachen, Pauwelsstr. 30

Priv.-Doz. Dr. med. Johannes Breuer

Oberarzt der Abt. Kinderheilkunde II. Universitätskinderklinik, Neuklinikum der Universität, 72076 Tübingen, Hoppe-Seyler-Str. 3

Dr. med. Thomas Bruhns

Abt. Kinderheilkunde II. Universitätskinderklinik, Neuklinikum der Universität, 72076 Tübingen, Hoppe-Seyler-Str. 3

Jutta Döttling-Ulrich

Dipl.-Sozialpädagogin (FH), Familientherapeutin, 72074 Tübingen, Gartenstr. 53/2 
Dr. med. Herbert Enders

Abteilung Klinische Genetik,

Institut für Anthropologie u. Humangenetik der Universität,

72074 Tübingen, Wilhelmstr. 27

Dr. med. Dieter Hassberg

Facharzt für Kinderkrankheiten-Kinderkardiologie,

72076 Tübingen, Waldhausen 15

Dr. med. Christoph Irtel von Brenndorff

Oberarzt der Abt. Kinderheilkunde II. Universitätskinderklinik,

Neuklinikum der Universität,

72076 Tübingen, Hoppe-Seyler-Str. 3

Prof. Dr. med. Hans-Heiner Kramer

Direktor der Klinik für Kinderkardiologie

und Biomedizinische Technik,

Christian-Albrechts-Universität zu Kiel,

24105 Kiel, Schwanenweg 20

Dr. med. Ferdinand T. Kuhn-Regnier

Oberarzt, Klinik und Poliklinik für Herz- und Thoraxchirurgie,

Medizin. Einrichtungen der Universität Köln,

50924 Köln, Joseph-Stelzmann-Str. 9

Priv.-Doz. Dr. med. G. Mielke

Universitäts-Frauenklinik,

72076 Tübingen, Schleichstr. 4

Prof. Dr. med. Heinrich Netz

Kinderkardiologie und pädiatrische Intensivmedizin,

Klinikum Großhadern,

81366 München

Prof. Dr. med. Werner Rosendahl

Nachsorgeklinik Tannheim GmbH,

78052 VS-Tannheim, Gemeindewaldstr. 75

Priv.-Doz. Dr. med. Ludger Sieverding

Oberarzt der Abt. Kinderheilkunde II. Universitätskinderklinik, Neuklinikum der Universität,

72076 Tübingen, Hoppe-Seyler-Str. 3

Prof. Dr. med. Helmut Singer

Pädiatrische Kardiologie der Klinik mit Poliklinik

für Kinder und Jugendliche der Univ. Erlangen-Nürnberg,

91054 Erlangen, Loschgestr. 15 
Prof. Dr. med. Achim A. Schmaltz Direktor der Abt. f. Kardiologie, Klinik und Poliklinik für Kinder- und Jugendmedizin, Zentrum für Kinderheilkunde, 45122 Essen, Hufelandstr. 55

Dr. med. Egon Steil

Oberarzt der Abt. Kinderheilkunde II. Universitätskinderklinik, Neuklinikum der Universität, 72076 Tübingen, Hoppe-Seyler-Str. 3

Dr. med. Thomas Teufel

71088 Holzgerlingen, Lichtensteinstraße 11/2

Prof. Dr. med. Herbert E. Ulmer

Ärztlicher Direktor der Abteilung Pädiatrische Kardiologie an der Univ.-Kinderklinik,

69120 Heidelberg, Im Neuenheimer Feld 153

Prof. Dr. med. Ernst-Rainer de Vivie

Direktor der Klinik und Poliklinik für Herz- und Thoraxchirurgie, Medizin. Einrichtungen der Universität Köln, 50924 Köln, Joseph-Stelzmann-Str. 9

Prof. Dr. med. Johannes Vogt

Direktor der Klinik und Poliklinik für Kinderheilkunde Kardiologie der Universität Münster, 48129 Münster, Albert-Schweitzer-Str. 33

Prof. Dr. med. Gisbert Wagner Direktor der Klinik und Poliklinik für Pädiatrische Kardiologie, Martin-Luther-Universität Halle-Wittenberg, 06120 Halle/Saale, Ernst-Grube Str. 40 